\title{
Canalicular lacerations: Causes, related ocular injury and management at St John Eye Hospital
}

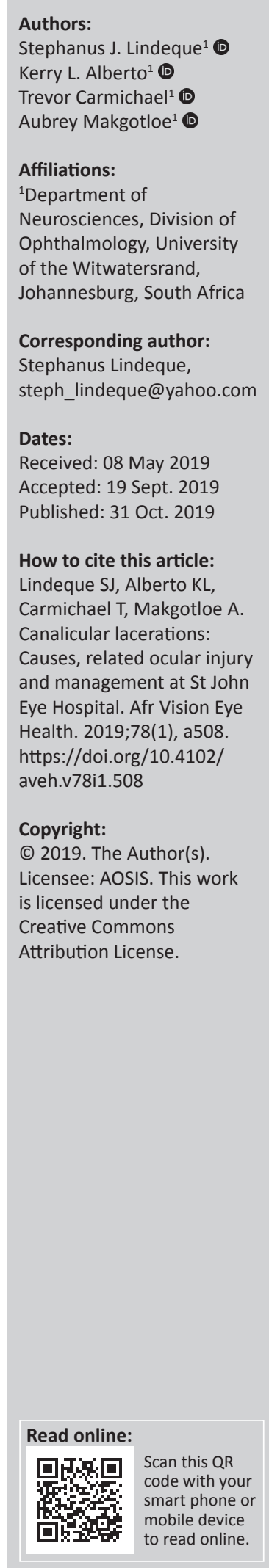

Background: Canalicular lacerations cause significant morbidity if unrepaired and may be associated with serious ocular injuries. Few studies describe the causes of these injuries, and none of these were conducted in Southern Africa.

Aim: The aim of this study was to describe the incidence of canalicular lacerations in terms of their causes, associated ocular injuries and management at St John Eye Hospital.

Setting: St John Eye Hospital, Soweto, Gauteng Province, South Africa.

Methods: This was a prospective descriptive study of all patients presenting with canalicular lacerations at St John Eye Hospital over a 7-month period. Data were collected from the patient files and analysed using descriptive statistics.

Results: Seventy-eight patients presented at St John Eye Hospital with lid lacerations. Of these 78 patients, $26(33.3 \%)$ had canalicular involvement. There was only mild male preponderance. The median age was 34 years. The most common cause of injury was assault (58\%) usually involving a knife. There were associated ocular injuries in $35 \%$ of the patients. Globe rupture was relatively common (8\%). All patients received lid repair under general anaesthetic. Additional surgical procedures were performed in 19\%. The median duration of surgery was 103 minutes. Canalicular repair consumed $100 \mathrm{~min}$ of operating time per week.

Conclusion: This study demonstrates that canalicular lacerations are relatively common at St John Eye Hospital. These are disproportionately caused by assault and more often associated with serious ocular injuries. This places considerable burden on already limited theatre resources and may be of particular relevance in the South African setting.

Keywords: canalicular lacerations; causes; associated injuries; management; South Africa; St John Eye Hospital; epidemiology.

\section{Introduction}

Canalicular lacerations commonly occur in cases of traumatic lid laceration ${ }^{1}$ and require prompt surgical repair ideally within 24 hours -48 hours. ${ }^{2}$ If not adequately repaired, these injuries may result in significant morbidity in the form of chronic epiphora. ${ }^{3}$ In addition, these injuries may be associated with significant trauma to the orbit and globe, requiring additional care. ${ }^{3}$

Canalicular lacerations either result from direct trauma, as with a sharp object, for example, blade or glass fragment, or indirectly because of lateral traction, such as that caused by blunt trauma at a point distant from the laceration. ${ }^{4}$

Studies involving the causes of canalicular lacerations are limited. ${ }^{3,5}$ Previous studies into the causes of canalicular lacerations have shown considerable variation in the mechanisms of injury in each region. These include lids hooking on garment fasteners, ${ }^{1}$ physical altercations,${ }^{5,6,7}$ falls, ${ }^{8}$ dog bites ${ }^{9}$ and motor vehicle collisions. ${ }^{10}$ The reason for this variation has been postulated, by Naik et al., to be because of socio-cultural differences amongst populations. ${ }^{1}$ Therefore, it is reasonable to expect that the most common causes of these injuries in South Africans cannot simply be inferred from other studies.

Laceration of the eyelid is a well-described complication of both blunt and sharp trauma to the face, ${ }^{11}$ and $16 \%$ of lacerated lids involve the canaliculi. ${ }^{3}$ The lower canaliculus is lacerated more often than is the upper canaliculus, ranging from $52 \%$ to $78.5 \%$, as opposed to $16.6 \%-32.0 \%$ for upper canalicular injuries. Both canaliculi are lacerated in $4.8 \%-15 \%$ of patients. These injuries tend to affect young patients more frequently, with the average age ranging from 16 to 39 years 
old. Men are disproportionately represented and form between $72.0 \%$ and $88.6 \%$ of presenting patients in the literature. ${ }^{1,3,5,6,6,8,9,10,12,13,14}$

Although no single cause has been consistently shown to be most common, certain mechanisms of injury do occur commonly in multiple studies. Frequently reported canalicular injuries in other studies are because of physical altercations, animal bites, falls, road accidents and sharp objects such as tree branches. ${ }^{6}$ Dog bites have been disproportionately found to affect children. A study found that dog bites were more likely to injure the canaliculi than other causes of lid injury. ${ }^{15}$

Findings of the respective studies are summarised in Table 1.

Canalicular lacerations are reportedly associated with other ocular injuries at a rate between $20 \%$ and $40 \%{ }^{13}$ These injuries tend to occur more often when blunt injury is the cause of the canalicular laceration. ${ }^{3}$ The frequency and nature of these injuries varies from study to study but common examples include commotio retinae, corneal abrasions, hyphaema, globe rupture, corneal laceration, scleral laceration, lens injury, vitreous haemorrhage, retinal haemorrhage, intraocular foreign body and traumatic optic neuropathy. $1,3,5,7,8,13$ Findings of the respective studies are summarised in Table 2.

The placement of a canalicular stent during reattachment of the severed canalicular ends has become widely accepted to be essential for successful outcomes. ${ }^{11}$ The principles of surgical repair are early anastomosis, stent placement and the use of magnification. ${ }^{16}$ Repair techniques may be classified as bicanalicular or monocanalicular. Bicanalicular stents may be nasally or annularly intubated.
Bicanalicular repair with nasal intubation is considered to be the gold standard for both monocanalicular and bicanalicular repair. ${ }^{17}$ It involves intubating both the injured and the uninjured canaliculus with a silicone tube, which is then fixated in the nose. General anaesthesia is recommended because of the painful nature of canalicular intubation into the nose and because patients tend to be young. ${ }^{11}$

Bicanalicular repair with retrograde probing and annular intubation involves the use of a curved metal probe (pigtail probe) to intubate both canaliculi in a ring-like fashion, under general anaesthetic. The risk of injuring the uninvolved canaliculus is increased when using this method, as false tracts may be created. As such, it is described as being best used as a last resort. ${ }^{17}$

Monocanalicular repair is accomplished using a silicone stent which is short and ends in a punctal plug. The stent is inserted into the punctum of the lacerated canaliculus and passed via the distal canalicular end into the lacrimal sac. This means there is no injury risk to the uninjured canaliculus. ${ }^{1}$ The most widely used of these stents is the Mini Monoka. ${ }^{1}$ Aside from not risking the other canaliculus, this technique has certain advantages over conventional bicanalicular stenting. It is less invasive ${ }^{17}$ is less technically challenging, has less complications and may be performed under local anaesthetic in a cooperative patient. ${ }^{5}$

Murchison et al. found no significant difference in the success rate of mono- and bicanalicular repairs, ${ }^{7}$ but did find that the training level of the surgeon significantly affected success for all types of repair. Furthermore, the success rate was much greater if the repair was performed in theatre, as opposed to a side room in casualty.

The aim of this study was to identify causes of canalicular laceration seen at St John Eye Hospital (SJEH), Soweto,

TABLE 1: Causes of canalicular lacerations in other studies.

\begin{tabular}{|c|c|c|c|c|c|}
\hline Author & Region & Sample size & Most common cause & Second most common cause & Third most common cause \\
\hline Jordan et al. ${ }^{3}$ & Nashville, United States & 236 & Sharp objects $(23 \%)$ & Dog bites $(20 \%)$ & $\begin{array}{l}\text { Blunt objects ( } 18 \%) \\
\text { Motor vehicle crashes }(18 \%)\end{array}$ \\
\hline Kennedy et al. ${ }^{9}$ & Philadelphia, United States & 222 & Body contact from another person (36\%) & Dog bites and scratches $(14 \%)$ & Falls $(15 \%)$ \\
\hline Wu et al. ${ }^{10}$ & Taoyuan City, Taiwan & 98 & Road accidents (43\%) & Falls $(15 \%)$ & Animal bites $(4 \%)$ \\
\hline Al-Batayneh et al. ${ }^{8}$ & Amman, Jordan & 44 & Falls $(61 \%)$ & Road accidents ( $16 \%)$ & Sharp object trauma (14\%) \\
\hline Sendul et al. ${ }^{5}$ & Istanbul/Kars/Konya, Turkey & 42 & Assault $(33 \%)$ & Road accidents $(24 \%)$ & Falls $(14 \%)$ \\
\hline
\end{tabular}

TABLE 2: Associated injuries of canalicular lacerations.

\begin{tabular}{|c|c|c|c|c|c|c|}
\hline Author & Region & $\begin{array}{l}\text { Sample } \\
\text { size }\end{array}$ & $\begin{array}{l}\text { Total incidence } \\
\text { of associated ocular } \\
\text { injuries }\end{array}$ & $\begin{array}{l}\text { Most common associated } \\
\text { ocular injury }\end{array}$ & $\begin{array}{l}\text { Second most common } \\
\text { associated ocular injury }\end{array}$ & $\begin{array}{l}\text { Third most common } \\
\text { associated ocular injury }\end{array}$ \\
\hline Jordan et al. ${ }^{3}$ & Nashville, United States & 236 & $6.4 \%$ & Globe rupture $(4.2 \%)$ & $\begin{array}{l}\text { Optic neuropathy }(0.8 \%) \\
\text { Retinal detachment } \\
(0.8 \%)\end{array}$ & - \\
\hline Kennedy et al. ${ }^{9}$ & Philadelphia, United States & 222 & Not specified & Commotio retinae $(12.2 \%)$ & Corneal abrasion (10.8\%) & Hyphaema $(6.8 \%)$ \\
\hline Murchison et al. ${ }^{7}$ & Philadelphia, United StatesA & 137 & $32.1 \%$ & Intraocular injuries $(19.7 \%)$ & $\begin{array}{l}\text { Ocular surface injuries } \\
(12.4 \%)\end{array}$ & - \\
\hline Sendul et al. ${ }^{5}$ & Istanbul/Kars/Konya, Turkey & 42 & $33.3 \%$ & $\begin{array}{l}\text { Conjunctival laceration } \\
(11.9 \%)\end{array}$ & Corneal abrasion $(9.5 \%)$ & Hyphaema $(4.8 \%)$ \\
\hline Naik et al. ${ }^{1}$ & Hyderabad, India & 24 & $25 \%$ & $\begin{array}{l}\text { Hyphaema }(8.3 \%) \\
\text { Intraocular foreign body }(8.3 \%)\end{array}$ & - & $\begin{array}{l}\text { Corneo-scleral laceration (4.2\%) } \\
\text { Conjunctival laceration }(4.2 \%)\end{array}$ \\
\hline
\end{tabular}


Gauteng Province, South Africa, as well as to describe the associated ocular injuries and the burden of management on theatre resources.

\section{Methodology}

This was a prospective study describing the causes of injury and associated injuries of all patients with canalicular laceration presenting at SJEH over a 7-month period. Furthermore, the surgical procedure performed and the theatre time consumed were recorded. St John Eye Hospital is the ophthalmic department of the Chris Hani Baragwanath Academic Hospital, which is the world's third largest hospital. It is located in Soweto, Gauteng Province, South Africa. The St John Eye Hospital services approximately 50000 ophthalmological patients annually. The hospital serves patients from Soweto, as well as patients from the surrounding Greater Johannesburg area. Furthermore, it functions as a tertiary referral centre for peripheral hospitals in Gauteng.

All patients who presented at SJEH, from 22 December 2017 to 22 July 2018, with a canalicular laceration were included in the study. Patients who presented in an unstable state and thus required urgent transfer to an emergency department for resuscitation were to be excluded.

Relevant data were collected from the files of patients. These included demographical data (age, sex and race), canaliculus involved, cause of injury, associated ocular injuries, surgical procedures performed, theatre time consumed and whether general anaesthetic was administered.

Data analysis was carried out using STATISTICA version 13.1. Categorical variables were summarised using frequency and percentage tabulation and illustrated by means of bar charts. Continuous variables were summarised by the mean, median and interquartile range, and their distribution was illustrated by means of histograms. Based on worst-case (for sample size) estimates of 50\%, 5\% precision and the $95 \%$ confidence level, a sample size of 385 would be required. The actual sample size of 26 in this study corresponds to a precision of $19.2 \%$ (rather than $5.0 \%$ ), which is a limitation of the study.

\section{Ethical considerations}

Permission was obtained from the Medical Advisory Committee of Baragwanath Hospital, the chief executive officer of Chris Hani Baragwanath Academic Hospital and the Clinical Head of Department at St John Eye Hospital. A protocol was approved by the Human Research Ethics Committee of the University of the Witwatersrand (Protocol reference number M171031). All participants provided formal informed consent in writing. This study was carried out in accordance with the Declaration of Helsinki.

\section{Results \\ Patient characteristics}

During the period under review, 78 patients presented with lid lacerations at SJEH. Of these patients, 26 were found to have canalicular lacerations (33.3\%). All patients who presented with canalicular lacerations consented to participate in the study.

Males accounted for 18 (69\%) of the patients in the study, and $25(96 \%)$ of the patients were of African descent. There was one Caucasian patient $(4 \%)$. The average patient age was 31.5 years. The median age of the patients was 34 years (interquartile range 25-39 years; range 10 months to 60 years). Children accounted for four (15\%) of the patients in this study.

\section{Cause of injury}

The predominant cause of injury was assault which accounted for $15(58 \%)$ of the 26 patients. The second most common cause was road accident which accounted for five patients (19\%), of which three were motor vehicle collisions and two were pedestrian vehicle collisions.

\section{Canaliculus involved}

A single canalicular laceration occurred in 23 patients (88\%), three patients $(12 \%)$ had both upper and lower canaliculi

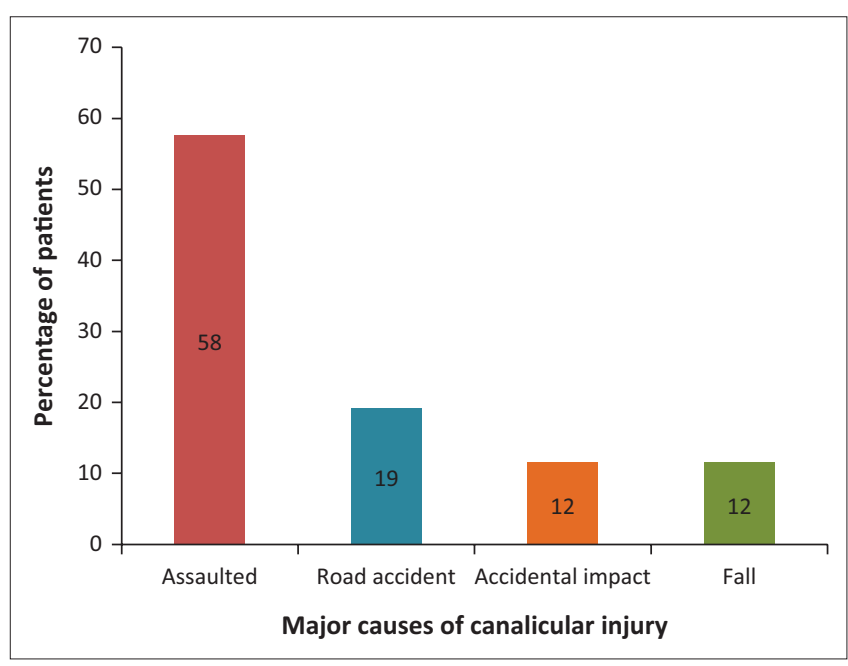

The most common type of assault was being stabbed with a knife. This cause accounted for four patients, which was $27 \%$ of all assaults and $15 \%$ of all canalicular lacerations. The average age of assaulted patients was 31.5 years (range 10 months to 44 years). FIGURE 1: Major causes of canalicular injury.

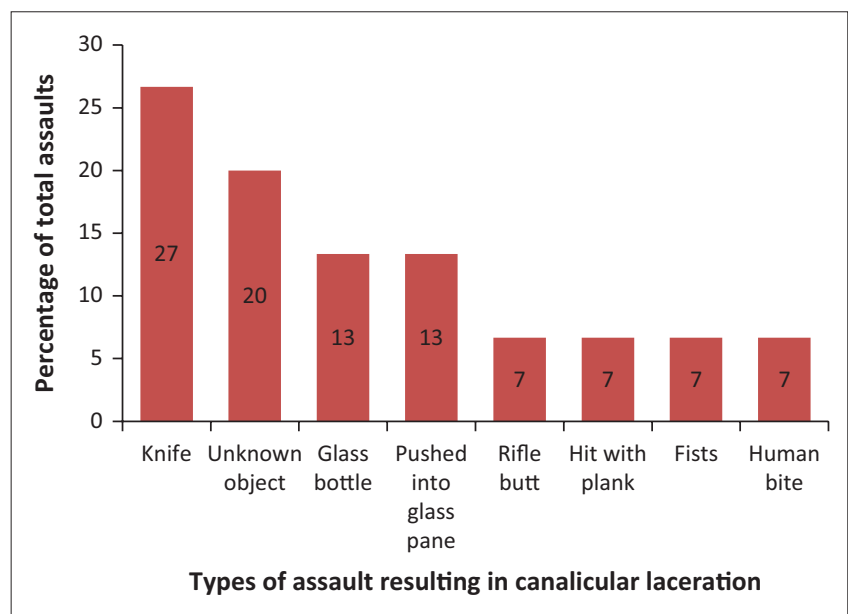

FIGURE 2: Types of assault resulting in canalicular laceration. 
lacerated. Lower canalicular lacerations accounted for 18 (62\%) of the 29 canalicular lacerations sustained by the 26 patients. The left lower canaliculus was the most frequently injured $(n=11 ; 42 \%)$, followed by the right lower $(n=7 ; 27 \%)$, the left upper $(n=6 ; 23 \%)$ and right upper canaliculus $(n=5$; $19 \%)$. The majority of patients sustained left-sided canalicular lacerations ( $n=15 ; 58 \%)$. Where assault was the cause, the left canaliculi were lacerated in eight patients and the right in seven patients $(53 \%$ vs. $47 \%)$. No bilateral canalicular lacerations were observed.

\section{Associated ocular injuries}

Associated ocular injuries were detected in nine patients (35\%). Traumatic uveitis $(n=4 ; 15 \%)$ and subconjunctival haemorrhage $(n=4 ; 15 \%)$ were the most common. Table 3 displays the incidence of each injury.

\section{Surgical management}

All patients received lid repair under general anaesthetic. Canalicular repair was successfully performed in 24 cases (92\%). Bicanalicular repair with nasal intubation was employed in 23 cases (88\%), while monocanalicular repair with a Mini Monoka tube was performed in one patient $(4 \%)$. Additional surgical procedures were performed for five patients (19\%); these are tabulated in Table 4.

\section{Duration of surgery}

A total of 2985 minutes ( $49 \mathrm{~h}$ and $45 \mathrm{~min}$ ) of general anaesthetic theatre time was consumed over the 7-month period. This amounted to $100 \mathrm{~min}$ per week. The average duration of surgery was $115 \mathrm{~min}$, while the median duration was $103 \mathrm{~min}$. The notable difference between average and mean duration is because of a single outlying case that consumed $265 \mathrm{~min}$. The range was $45 \mathrm{~min}-265 \mathrm{~min}$ with an interquartile range of $90 \mathrm{~min}-135 \mathrm{~min}$. The average duration for patients only comprising of lid and canalicular repair was $101 \mathrm{~min}$.

TABLE 3: Associated ocular injuries.

\begin{tabular}{|c|c|c|}
\hline Associated ocular injury & $a$ (total = 26) & Percentage \\
\hline Nil & 17 & 65 \\
\hline Traumatic uveitis & 4 & 15 \\
\hline $\begin{array}{l}\text { Subconjunctival } \\
\text { haemorrhage }\end{array}$ & 4 & 15 \\
\hline Conjunctival laceration & 1 & 4 \\
\hline Retrobulbar haemorrhage & 1 & 4 \\
\hline Globe rupture & 2 & 8 \\
\hline Medial rectus avulsion & 1 & 4 \\
\hline Optic nerve avulsion & 1 & 4 \\
\hline \multicolumn{3}{|c|}{$\begin{array}{l}\text { Note: Percentages do not sum to } 100 \% \text { because some patients had more than one ocul } \\
\text { injury. }\end{array}$} \\
\hline Additional surgical procedure & $n$ (Total $=26$ ) & Percentage \\
\hline Evisceration with silicone ball implant & 2 & 8 \\
\hline Skin graft & 1 & 4 \\
\hline Brow repair & 1 & 4 \\
\hline Orbital floor repair & 1 & 4 \\
\hline
\end{tabular}

\section{Discussion}

During this study, patients presenting with lid lacerations had more than double the canalicular involvement rate of $16 \%$ reported in the literature. ${ }^{3}$ Had this incidence rate continued, it would have translated to 45 canalicular lacerations per annum at SJEH. This is a relatively high incidence. Jordan et al. have published the largest study on the subject and reported an incidence of 28 canalicular lacerations per annum. ${ }^{3}$ These injuries may therefore represent a significant public health challenge in our setting.

Patients in his study tended to be older than those reported in other studies. ${ }^{1,3,5,6,7,8,9,10,12,13,14}$ This is contrary to the notion that these injuries affect mostly young patients, and indeed, the ages of patients in this study ranged from as young as 10 months to as old as 60 years. The relatively low number of children in this study may explain the relatively low incidence of dog bites as a cause of injury, as dog bites are known to disproportionately affect children. ${ }^{15}$

The expected male preponderance, while present, was lower than that reported by any other study we could find. $1,3,5,6,7,8,9,10,12,13,14$ This is unexpected, especially when considering that assault was by far the most common cause of injury.

The causes of injury reported in this study were similar in type to those reported in other studies, but the proportions were markedly different. $1,3,5,7,8,9,10$ More than half of the patients in this study (58\%) reported assault as the cause of their injuries. This cause of injury is also common in other studies, but the highest proportion reported was 36\%. ${ }^{9} \mathrm{Knife}-$ related assault was an especially common cause of injury in this study and accounted for $12 \%$ of all patients. A number of assaults involved a glass bottle as the weapon. This mode of injury is not specifically reported in other studies. It is worth bearing in mind that one in five assaulted patients could not identify the object used in the assault, and as such, these estimates of knife and glass bottle assault are likely conservative.

The left canaliculus was more often lacerated in this study than the right ( $58 \%$ vs. $42 \%$ ). It is tempting to attribute this difference to right-handed attackers, but it is not supported by the data. Only $53 \%$ of patients who reported assault as the cause of injury had a left-sided canalicular laceration. The left lower canaliculus was involved almost twice as any other canaliculus being injured in $42 \%$ of patients. The reason for this is unclear.

Associated ocular injuries were found in 35\% of patients, which is in keeping with a rate considered normal in the literature $(20 \%-40 \%) .{ }^{13}$ It is worth noting, however, that the author could find no study with a higher rate than 35\%. The majority of these associated ocular injuries tended to be mild, the most common injuries being traumatic uveitis and subconjunctival haemorrhage. However, $8 \%$ of patients suffered globe rupture necessitating evisceration. This is nearly double the rate observed by Jordan et al. in the largest 
study conducted on this subject, a study where globe rupture is reported as the most common associated ocular injury. ${ }^{3}$ This relatively high incidence of often serious injuries represents a considerable source of morbidity, as well as an additional burden on service delivery.

Despite resource limitations at $\mathrm{SJEH}$, all patients received lid repair under general anaesthetic and canalicular repair in keeping with international standards. Providing this essential service comes at the cost of cancelling important elective surgeries, as there is currently no dedicated ophthalmic trauma theatre at SJEH.

The average duration of surgery was $115 \mathrm{~min}$, but this is likely an overestimation as there was a single long outlying case $(265 \mathrm{~min})$. The median duration of $103 \mathrm{~min}$ is likely a better measure of resource burden, and this correlates well with the average duration of surgery for cases where only canalicular laceration repair was required of $101 \mathrm{~min}$.

Over the 7-month period, canalicular laceration repair was demonstrated to consume $100 \mathrm{~min}$ of general anaesthetic theatre time every week at SJEH. This was purely the time spent operating and did not take into account the time spent inducing and reversing anaesthesia, as well as cleaning and draping of the patient. An audit of time consumed in theatre at a teaching hospital in Nigeria found that the average surgical duration of an ophthalmological case was $25.7 \mathrm{~min} .{ }^{18}$ If the average surgical time per ophthalmological case at SJEH is similar to this, this would amount to 202 patients every year not receiving their scheduled eye surgery. Funding a dedicated ophthalmic trauma theatre may alleviate this burden.

This study suggested that canalicular lacerations may represent an especially pronounced public health challenge in the South African context. The study was limited by its single-centre design, small sample size and relatively short data collection period. As there is a paucity of similar studies locally, multicentre studies with longer data collection periods are required to verify these findings.

\section{Conclusion}

This study demonstrates that canalicular lacerations are relatively common at SJEH. These are disproportionately caused by assault and more often associated with serious ocular injuries. This places considerable burden on already limited theatre resources and may be of particular relevance in the South African setting.

More study is needed to verify these findings and identify potential strategies for primary prevention.

\section{Acknowledgements}

The authors would like to thank the staff of St John Eye Hospital. This research represents Dr S.J. Lindeque's, Master of Medicine at the University of the Witwatersrand. The final thesis has yet to be formally submitted for evaluation.

\section{Competing interests}

The authors declare that they have no financial or personal relationships that may have inappropriately influenced them in writing this article.

\section{Authors' contributions}

S.J.L. was the principal author and concept originator, and was responsible for the protocol design, data collection and analysis, literature review and discussion. K.L.A. was responsible for the supervision, concept review, protocol design revision and final approval. T.C. was responsible for the co-supervision and concept review. A.M. was responsible for the critical revision and final approval.

\section{Funding information}

This research was funded by the principal author.

\section{Data availability statement}

Data collected during this study are available from the corresponding author upon request via email.

\section{Disclaimer}

The views expressed in the submitted article are those of the authors and not an official position of the institution.

\section{References}

1. Naik MN, Kelapure A, Rath $\mathrm{S}$, et al. Management of canalicular lacerations: Epidemiological aspects and experience with Mini-Monoka monocanalicular stent. Am J Ophthal. 2008;145(2):375-380. https://doi.org/10.1016/j.ajo.2007.09.018

2. Bowling, B. Kanski's clinical ophthalmology: A systematic approach. 8th ed. Amsterdam: Elsevier, 2016; p. 863.

3. Jordan DR, Ziai S, Gilberg SM, et al. Pathogenesis of canalicular lacerations. Ophthalmic Plast Reconstr Surg. 2008;24(5):394-398. https://doi.org/10.1097/ IOP.0b013e318183267a

4. American Academy of Ophthalmology. Basic and clinical science course Section 7: Orbit eyelids, and lacrimal system. San Francisco, CA: American Academy of Ophthalmology, 2014; p. 272.

5. Sendul SY, Cagatay HH, Dirim B, et al. Reconstructions of traumatic lacrimal canalicular lacerations: A 5 years experience. Open Access J Sci Technol. 2015;3(1):1-6. https://doi.org/10.11131/2015/101121

6. Reifler DM. Management of canalicular laceration. Survey Ophthalmol. 1991;36(2):113-132. https://doi.org/10.1016/0039-6257(91)90125-Y

7. Murchison AP, Bilyk JR. Canalicular laceration repair: An analysis of variables affecting success. Ophthalmic Plast Reconstr Surg. 2014;30(5):410-414. https:// doi.org/10.1097/IOP.0000000000000133

8. Al-Batayneh I, Odat TA, Al-Dhoun A, et al. Incidence and management techniques of severed lacrimal canaliculi: 11 years experience. J R Med Serv. 2016:23(1):47-53. https://doi.org/10.12816/0023360

9. Kennedy RH, May J, Dailey J, et al. Canalicular laceration: An 11-year epidemiologic and clinical study. Ophthalmic Plast Reconstr Surg. 1990;6(1):46-53. https://doi. org/10.1097/00002341-199003000-00008

10. Wu SY, Ma L, Chen RJ, et al. Analysis of bicanalicular nasal intubation in the repair of canalicular lacerations. Jpn J Ophthalmol. 2010;54(1):24-31. https://doi. org/10.1007/s10384-009-0755-7

11. Kersten RC, Kulwin DR. 'One-stitch' canalicular repair: A simplified approach for repair of canalicular laceration. Ophthalmology. 1996;103(5):785-789. https:// doi.org/10.1016/S0161-6420(96)30615-5

12. Jordan DR. Monocanalicular lacerations: To reconstruct or not? Can J Ophthalmol. 2002;37(4):245-246. https://doi.org/10.1016/S0008-4182(02)80116-8 
13. Lee $\mathrm{H}$, Chi M, Park M, et al. Effectiveness of canalicular laceration repair using monocanalicular intubation with Monoka tubes. Acta Ophthalmol. 2009;87(7):793-796. https://doi.org/10.1111/j.1755-3768.2009.01644.x

14. Leibovitch I, Kakizaki H, Prabhakaran V, et al. Canalicular lacerations: Repair with the Mini-Monoka ${ }^{\circledR}$ monocanalicular intubation stent. Ophthalmic Surg Lasers Imaging Retina. 2010;41(4):472-477. https://doi.org/10.3928/15428877-20100525-05

15. Savar A, Kirszrot J, Rubin PA. Canalicular involvement in dog bite related eyelid lacerations. Ophthalmic Plast Reconstr Surg 2008;24(4):296-298, https://doi. org/10.1097/IOP.0b013e318177e21f
16. MacGillivray RF, Stevens MR. Primary surgical repair of traumatic lacerations of the lacrimal canaliculi. Oral Surg Oral Med Oral Pathol Oral Radiol Endod. 1996;81(2):157-163. https://doi.org/10.1016/S1079-2104(96)80406-9

17. Black EH, Nesi FA, Calvano CJ, et al. Smith and Nesi's ophthalmic plastic and reconstructive surgery. New York: Springer Science \& Business Media, 2012; p. 664-667.

18. Mpyet CD. An audit of the use of ophthalmic theatre time. Community Eye Health. 2002;15(44):62. 